\title{
A prospective randomized clinical study evaluating the efficacy and compliance of oral sulfate solution and 2-L ascorbic acid plus polyethylene glycol
}

Ki Hwan Kwon ${ }^{1,}$, Ji Ae Lee ${ }^{2,}$, Yun Jeong Lim ${ }^{1,}$ Beom Jae Lee ${ }^{2}$, Moon Kyung Joo², Yu Ra Sim², Wonjae Choi ${ }^{2}$, Taehyun $\mathrm{Kim}^{2}$, Ji Yoon Kim ${ }^{1}$, Ei Rie Cho ${ }^{1}$, Yoon Tae Jeen ${ }^{3}$, and Jong-Jae Park ${ }^{2}$

\begin{abstract}
${ }^{1}$ Department of Internal Medicine, Dongguk University Ilsan Hospital, Goyang; ${ }^{2}$ Department of Internal Medicine, Korea University Guro Hospital, Seoul; ${ }^{3}$ Department of Internal Medicine, Korea University Anam Hospital, Seoul, Korea
\end{abstract}

Received: August 7, 2017

Revised : November 17, 2017

Accepted: October 13, 2018

\section{Correspondence to}

Yun Jeong Lim, M.D.

Division of Gastroenterology,

Department of Internal

Medicine, Dongguk University

Ilsan Hospital, 27 Dongguk-ro,

Ilsandong-gu, Goyang 10326, Korea

Tel: +82-31-961-7133

Fax: +82-31-961-9339

E-mail: drlimyj@gmail.com

Jong-Jae Park, M.D.

Division of Gastroenterology,

Department of Internal

Medicine, Korea University

Guro Hospital, 148 Gurodong-

ro, Guro-gu, Seoul o8308, Korea

Tel: +82-2-2626-1771

Fax: +82-2-2626-2024

E-mail:gi7pjj@korea.ac.kr

*These authors contributed equally to this work.
Background/Aims: Oral sulfate solution (OSS) is an emerging cleansing agent for bowel preparation. However, data comparing OSS to other conventional bowel preparations in Asian patients are limited. Therefore, the objective of this study was to compare the efficacy and tolerability of OSS to ascorbic acid plus polyethylene glycol (AA + PEG) in Asian patients.

Methods: This was a prospective, randomized, parallel, investigator-blind study performed in two university hospitals in Korea. Bowel preparation efficacy was evaluated using both the Ottawa Bowel Preparation Scale (OBPS) and Boston Bowel Preparation Scale (BBPS).

Results: Among 173 patients, 86 received OSS while 87 received AA + PEG for bowel preparation. Total OBPS score was $2.80 \pm 2.48$ in the OSS group and $4.49 \pm 3.08 \mathrm{in}$ the AA + PEG group, indicating significantly $(p<0.001)$ better efficacy with OSS. Total BBPS was higher in the OSS group (7.43 \pm 1.49 vs. $6.51 \pm 1.76, p<0.001)$, indicating superior bowel preparation quality with OSS. Preparation-related adverse events were generally acceptable. Patients receiving OSS had more nausea (1.92 \pm 0.94 vs. $1.54 \pm 0.76, p=0.004)$ and abdominal cramping ( $1.45 \pm 0.78$ vs. $1.17 \pm 0.51, p=$ $0.006)$ than those receiving AA + PEG. However, overall satisfaction and taste were similar between the two groups.

Conclusions: OSS had a non-inferior bowel cleansing efficacy than AA + PEG regardless of colon segment.

Keywords: Ascorbic acid; Bowel preparation; Colonoscopy; Oral sulfate solution; Polyethylene glycols

\section{INTRODUCTION}

Adequate bowel preparation is essential for effective colonoscopies [1-5]. Poor bowel preparation results in missed polyps, increased risk of procedural adverse events, and the necessity for repeated examinations $[2,5]$. An ideal bowel cleansing agent should rapidly empty the fecal material without causing mucosal alteration of the 
colon or causing electrolyte imbalance. In addition, it should be tolerable and easy to consume $[1,2,4]$. No available agent satisfies all these characteristics.

High-volume (4-L) polyethylene glycol (PEG) has been widely used for bowel preparation because of its cleaning efficacy and safety [6]. However, some patients do not fully consume the preparation because of the large volume and poor palatability [1]. Low-volume bowel preparations seek to improve patient tolerability. Two-liter PEG with additional ascorbic acid as a laxative (AA + PEG) has similar efficacy compared to 4 -L PEG $[7,8]$. AA + PEG is safe for most patients except for those who are deficient in glucose-6-phosphate dehydrogenase [8,9].

In 2009 , a new osmotic preparation termed oral sulfate solution (OSS) was developed for bowel cleansing [10]. This hyperosmolar preparation does not cause significant fluid or electrolyte shifts possibly because sulfate is a poorly absorbed anion. The preparation regimen involves consumption of a reduced volume (946 $\mathrm{mL}$ ) of the preparation solution and 1,892 $\mathrm{mL}$ of water with additional fluid consumption permissible $[1,11]$.

Recent studies have demonstrated the efficacy of OSS compared to conventional bowel cleansing agents and shown comparable or better efficacy with an acceptable safety profile [10-15]. Three of these studies have compared the efficacy of OSS with AA + PEG using a non-inferiority design. However, their study subjects were Americans [11,12,14]. Such data for Asians are lacking.

Thus, the objective of this randomized, prospective, investigator-blind, parallel study was to compare the efficacy and tolerability of OSS to AA + PEG in Asian patients. Both preparations were in low-volume and given as split doses according to the Korea and United States Food and Drug Administration approved regimens. Outcomes included effectiveness in bowel cleansing, tolerability, and compliance with the preparation.

\section{METHODS}

\section{Overview}

This was a prospective, randomized, parallel, investigator-blind study of adult patients undergoing elective colonoscopy. Subjects were enrolled from February 1st, 2016 to July 27th, 2016 at two university hospitals in Korea. Both study centers used the same investigation- al protocols. Subjects received OSS or AA + PEG bowel preparation. A 1:1 ratio with an almost equal distribution of patients was used in each study center. Colonoscopists were unaware of bowel preparation agent used for the patient to ensure that treatment blinding was maintained.

Subjects were patients aged 20 to 70 years who were undergoing screening colonoscopy. Subjects were excluded if they met any of the following criteria: (1) active colitis, suspicion of bowel obstruction or bleeding, (2) severe constipation, (3) ascites, (4) inflammatory bowel disease, (5) previous bowel surgery, (6) heart failure or ischemic heart disease within 6 months prior to enrollment, (7) renal insufficiency, (8) pregnant or breastfeeding, or (9) diagnosis of hypersensitivity. The consent form was approved by the Institutional Review Board of each study center (IRB No. DUIH2015-130, KUGH15296). Written informed consent was obtained from each participating patient.

\section{Administration of study agents}

After assignment of bowel preparation agents, subjects consumed a low-residue diet until three days before the colonoscopy. Before the diet, they received information regarding unacceptable foods, including vegetables or grains containing high dietary fiber, fruits containing seeds, seaweed, dry beans, and nuts. All subjects were instructed to fast from the evening before the day of the procedure.

OSS (Innofree, MH Healthcare, Seoul, Korea) contained $17.5 \mathrm{~g}$ sodium sulfate, $3.13 \mathrm{~g}$ potassium sulfate, 1.6 $\mathrm{g}$ magnesium sulfate, and flavoring agents in an aqueous liquid form supplied in a $177 \mathrm{~mL}$ bottle. Contents of each bottle were diluted with water to $473 \mathrm{~mL}$. Each subject was prescribed two bottles of OSS for bowel preparation. One bottle of OSS solution was diluted with water $(946 \mathrm{~mL})$ and consumed the evening before the day of the colonoscopy. The second bottle of diluted OSS was consumed the morning of the colonoscopy at least 3 hours prior to the procedure.

AA+PEG (Coolprep, Taejoon Pharm Co., Seoul, Korea) contained (per L) $100 \mathrm{~g}$ PEG, $1 \mathrm{~g}$ potassium chloride, 2.69 g sodium chloride, $7.5 \mathrm{~g}$ sodium sulfate, $4.7 \mathrm{~g}$ ascorbic acid, and 5.9 g sodium ascorbate. Subjects who were assigned with AA + PEG were instructed to ingest $1 \mathrm{~L}$ of AA + PEG solution and a further $500 \mathrm{~mL}$ water in the eve- 
ning before the day of the procedure. Then they began to consume the remaining $1 \mathrm{~L}$ of AA + PEG solution with water 5 hours prior to the procedure. The consumption should be completed at least 3 hours before the colonoscopy. All patients were instructed to return any remaining bowel preparation.

\section{Assessments of bowel preparation quality}

All study procedures were performed by expert colonoscopists who were blinded to bowel preparation agent. They evaluated the efficacy of bowel cleansing using the Ottawa Bowel Preparation Scale (OBPS) and Boston Bowel Preparation Scale (BBPS) [16,17]. OBPS assesses cleanliness and fluid volume separately. Cleanliness was assessed for the right colon (cecum, ascending), middle colon (transverse, descending), and the rectosigmoid colon. Each colon section was individually rated from o to 4 (o, no liquid; 1 , minimal liquid with no suctioning required; 2, suction required to view mucosa; 3, wash and suction; 4, solid stool, not washable). Fluid quantity was rated from o to 2 for the entire colon (o, minimal; 1, moderate; 2, large). OBPS ranged from o (perfect) to 14 (solid stool in each colon segment and copious fluid; i.e., a completely unprepared colon) [17]. Adequate bowel preparation was defined as all scores of each section $\leq 3$ and fluid quantity $<2[18]$.

BBPS assessed cleanliness for the right colon (cecum, ascending), transverse colon (transverse, hepatic, and splenic flexure), and the left colon (descending, rectosigmoid colon). Each colon section was individually rated from o to 3 (o, unprepared colon segment with mucosa not observed due to solid stool that cannot be cleared; 1, portion of mucosa of the colon segment observed but other areas of the colon segment not well observed due to staining, with residual stool and/or opaque liquid; 2, minor amount of residual staining, small fragments of stool and/or opaque liquid but mucosa of colon segment well observed; 3, entire mucosa of colon segment well observed with no residual staining, small fragments of stool, or opaque liquid). BBPS ranged from o (completely unprepared colon) to 9 (perfect). Adequate bowel preparation was defined as all scores of each section $\geq 2$ [16].

Investigators participating in this study were instructed on how to assess the bowel preparation scale. A complete colonoscopy was successful cecal intubation by visualization of the ileocecal valve and appendiceal orifice.

\section{Assessments of tolerability and adverse events}

After completing the assigned preparation and before the colonoscopy, all subjects completed a questionnaire on compliance (complete intake rate), overall tolerability, taste, abdominal cramping, stomach bloating, nausea, sleep disturbance, and intention to re-intake the same agent. Patients used a 5-point scale for each system (1, none; 2 , mild; 3 , bothersome; 4 , distressing; and 5 , severely distressing). This symptom scoring system has been used in previous studies [12,14]. If serious adverse events occurred, physicians assessed symptoms and signs. Additional evaluations were done.

\section{Sample size and statistical analyses}

A hypothesis of no difference between the two treatments in the overall quality of bowel cleansing was postulated. A non-inferiority design was applied. Non-inferiority was defined if the lower limit of the one-sided $97.5 \%$ confidence interval for the difference in success rates between the two treatment groups was $<15 \%$. At least 80 patients were required for each treatment group to detect a difference in treatment success with $5 \%$ type-I error rate and $80 \%$ power for a two-tailed chisquare test. Our sample size was based on results from previous studies with PEG solution $[1,2]$. Based on these data, we decided to enroll at least 90 patients per treatment group for this study. SPSS version 23 (IBM Co., Armonk, NY, USA) was used for all data analyses. Continuous variables were compared by the two-sided $t$ test while categorical variables were compared by Pearson's chi-square test or Fisher's exact test. A p value $<0.05$ was considered statistically significant.

\section{RESULTS}

\section{Demographics}

Initially, 187 subjects were randomized to either of the two bowel preparation regimens. Fourteen subjects were excluded, including one who met the exclusion criteria and 13 who withdrew consent before colonoscopy. Finally, 173 subjects were enrolled, including 86 subjects who received OSS and 87 subjects who received AA + PEG. Demographics and colonoscopy results of these two groups are presented in Table 1. Cecal intubation rate was $100 \%$ in both groups. Both groups were similar 
Table 1. Demographic, clinical, and endoscopic characteristics of subjects

\begin{tabular}{|c|c|c|c|}
\hline Characteristic & $\operatorname{OSS}(\mathrm{n}=86)$ & $\mathrm{AA}+\mathrm{PEG}(\mathrm{n}=87)$ & $p$ value \\
\hline Age, yr & $53.57 \pm 10.99$ & $56.22 \pm 10.54$ & 0.11 \\
\hline Male sex & $37(43.0)$ & $39(44.8)$ & 0.88 \\
\hline Body mass index, kg/m² & $23.70 \pm 3.00$ & $23.90 \pm 3.77$ & 0.71 \\
\hline Current smoking & $18(20.9)$ & $19(21.8)$ & 1.00 \\
\hline Diabetes & $8(10.1)$ & $8(9.2)$ & 1.00 \\
\hline Hypertension & $20(23 \cdot 3)$ & $23(26.4)$ & 0.73 \\
\hline Previous colonoscopy & $62(74 \cdot 4)$ & $63(72.4)$ & 0.86 \\
\hline Diet restriction & $66(76.7)$ & $75(86.2)$ & 0.12 \\
\hline Interval between the last ingestion and the colonoscopy, hr & $3.26 \pm 0.27$ & $3.34 \pm 0.32$ & 0.10 \\
\hline Cecal intubation time, sec & $240 \pm 122$ & $246 \pm 161$ & 0.78 \\
\hline
\end{tabular}

Values are presented as mean \pm SD or number (\%).

OSS, oral sulfate solution; AA + PEG, ascorbic acid plus polyethylene glycol.

Table 2. Outcome of bowel cleansing according to Ottawa Bowel Preparation Scale

\begin{tabular}{lccc}
\hline Variable & OSS $(\mathrm{n}=86)$ & AA + PEG $(\mathrm{n}=87)$ & $p$ value \\
\hline Total score & $2.80 \pm 2.48$ & $4.49 \pm 3.08$ & $<.001$ \\
$\quad$ Right colon & $0.73 \pm 0.95$ & $1.37 \pm 1.15$ & $<.001$ \\
Middle colon & $0.69 \pm 0.94$ & $1.24 \pm 1.08$ & $<0.001$ \\
Rectosigmoid colon & $0.83 \pm 0.96$ & $1.30 \pm 1.06$ & 0.002 \\
Quantity of fluid & $0.64 \pm 0.51$ & $0.76 \pm 0.55$ & 0.140 \\
Adequate bowel preparation & $80(93.0)$ & $67(77.0)$ & 0.005 \\
\hline
\end{tabular}

Values are presented as mean \pm SD or number (\%). Adequate bowel preparation was defined as all scores of each section $\leq 2$ and fluid quantity $\leq 1$.

OSS, oral sulfate solution; AA + PEG, ascorbic acid plus polyethylene glycol.

in gender, age, body mass index, smoking history, diet restriction, interval between the last ingestion and the colonoscopy, and cecal intubation time. The average age of study participants was $54.9 \pm 10.8$ years (range, 25 to 76). Diet restriction was undertaken in $76.7 \%$ (66/86) of the OSS group and $86.2 \%$ (75/87) of the AA + PEG patients $(p=0.12)$. Mean interval time between the end of the last purgative ingestion and the start of colonoscopy was $3.26 \pm 0.27$ hours for the OSS group and $3.34 \pm$ 0.32 hours for the AA + PEG group. Overall mean cecal intubation time was $243 \pm 143$ seconds without showing significant group difference $(240 \pm 122$ seconds for OSS vs. $246 \pm 161$ seconds for $\mathrm{AA}+\mathrm{PEG}, p=0.78)$.

\section{Efficacy of bowel cleansing}

Table 2 presents OBPS cleansing scores for the two groups. The rate of adequate bowel preparation was
93.0\% in the OSS group and $77.0 \%$ in the AA + PEG group $(p=0.005)$. Total OBPS score was $2.80 \pm 2.48$ in the OSS group, which was significantly $(p<0.001)$ lower (i.e., better) than $(4.49 \pm 3.08)$ of the AA + PEG group. Scores for the right, middle, and rectosigmoid colon segments were also significantly lower in the OSS group than THOSE in the AA + PEG group. However, quantities of fluids were comparable between the two groups ( $0.64 \pm 0.51$ in the OSS group vs. $0.76 \pm 0.55$ in the AA + PEG group, $p=0.14$ ). Results of comparison of bowel preparations based on BBPS are presented in Table 3. The rate of adequate bowel preparation was not significantly different between OSS and AA + PEG groups (86.0\% vs. 73.6\%, $p=0.06$ ). However, total BBPS score was significantly higher (i.e., better) in the OSS group ( $7.43 \pm 1.49$ vs. $6.51 \pm 1.76, p<0.001)$. Scores for the right, transverse, and left colon sections were consistently and 
Table 3. Outcome of bowel cleansing according to Boston Bowel Preparation Scale

\begin{tabular}{lccc}
\hline Variable & OSS $(\mathrm{n}=86)$ & AA + PEG $(\mathrm{n}=87)$ & $p$ value \\
\hline Total score & $7.43 \pm 1.49$ & $6.51 \pm 1.76$ & $<.001$ \\
Right colon & $2.36 \pm 0.68$ & $2.08 \pm 0.71$ & 0.009 \\
Transverse colon & $2.52 \pm 0.59$ & $2.23 \pm 0.74$ & 0.005 \\
Left colon & $2.55 \pm 0.59$ & $2.21 \pm 0.73$ & 0.001 \\
Adequate bowel preparation & $74(86.0)$ & $64(73.6)$ & 0.060 \\
\hline
\end{tabular}

Values are presented as mean \pm SD or number (\%). Adequate bowel preparation was defined as all scores of each section $\geq 2$. OSS, oral sulfate solution: AA + PEG, ascorbic acid plus polyethylene glycol.

Table 4. Compliance and adverse effects of bowel preparation regimen

\begin{tabular}{lccc}
\hline Variable & OSS $(\mathrm{n}=86)$ & AA + PEG $(\mathrm{n}=87)$ & $p$ value \\
\hline Complete intake rate & $82(95.3)$ & $82(94.3)$ & 1.000 \\
Overall satisfaction $^{\mathrm{a}}$ & $2.01 \pm 1.09$ & $1.98 \pm 0.98$ & 0.826 \\
Intention to re-intake $^{\mathrm{a}}$ & $69(80.2)$ & $72(82.8)$ & 0.699 \\
Taste $^{\mathrm{a}}$ & $1.98 \pm 1.07$ & $1.83 \pm 0.93$ & 0.330 \\
Nausea $^{\mathrm{a}}$ & $1.92 \pm 0.94$ & $1.54 \pm 0.76$ & 0.004 \\
Stomach bloating $^{\mathrm{a}}$ & $1.98 \pm 1.04$ & $1.76 \pm 0.88$ & 0.137 \\
Abdominal cramping $^{\mathrm{a}}$ & $1.45 \pm 0.78$ & $1.17 \pm 0.51$ & 0.006 \\
Sleep disturbance $^{\mathrm{a}}$ & $1.28 \pm 0.73$ & $1.21 \pm 0.57$ & 0.471 \\
\hline
\end{tabular}

Values are presented as number (\%) or mean \pm SD.

OSS, oral sulfate solution: AA + PEG, ascorbic acid plus polyethylene glycol.

${ }^{\mathrm{a}}$ Ratings, 1 = none; to $5=$ severely distressing.

significantly higher in the OSS group than those in the $\mathrm{AA}+\mathrm{PEG}$ group.

\section{Compliance, safety, and tolerability}

Data collected from the patient questionnaire about the compliance and tolerability of the bowel preparation are presented in Table 4. Among a total of 173 subjects, 164 subjects $(94.7 \%)$ ingested the full dosage regimen. Nine subjects (5.2\%) ingested more than half the dose regimen, but not the full dosage. There was no significant difference in overall satisfaction between OSS and AA + PEG groups $(2.01 \pm 1.09$ vs. $1.98 \pm 0.98$, $p=0.826$ ).

No serious adverse effects developed after ingestion of either preparation regimen. The OSS group had significantly higher (i.e., worse) scores for nausea (1.92 \pm 0.94 vs. $1.54 \pm 0.76, p=0.004)$ and abdominal cramping ( $1.45 \pm$ 0.78 vs. $1.17 \pm 0.51, p=0.006$ ). However, average scores for both groups were $<2$ (mild). Significant differences for taste, stomach bloating, and sleep disturbance between the two groups were not evident. Intention to re-intake the same preparation for future colonoscopies was similar in both groups.

\section{DISCUSSION}

We described a prospective, randomized, parallel, investigator-blind trial that compared the efficacy, safety, and tolerability between OSS and AA + PEG bowel preparations. This is the first report to analyze the efficacy and acceptability of OSS formulation in Korean subjects. Clinical outcomes were collected from 173 patients at university hospitals in Korea during screening colonoscopies. We demonstrated that OSS was superior to AA + PEG for colon cleansing. Tolerability and adverse events associated with these two preparations were similar except for nausea and abdominal cramping. However, these adverse events were generally acceptable. 
A previous study has shown that OSS provides superior segmental cleansing compared to 4 L PEG [13]. However, OSS was not directly compared to $4 \mathrm{~L}$ split-dose PEG [13]. Cleansing efficacies were worse if subjects received full AA + PEG dose in the evening before the procedure compared to split-dose [7]. In this study, splitdose regimens of OSS and AA + PEG were implemented to provide more successful preparation efficacy and less gastrointestinal adverse events compared to the sameday regimen [11].

Previous studies have frequently used OBPS and BBPS bowel cleansing scoring systems $[16,17]$. The BBPS is a valid and reliable measure of bowel preparation that aggregates scores of three broad segments of the colon rated during withdrawal of the colonoscope after washing and suctioning of the fluid. Consequently, this scoring system does not include the quantity of fluid in the colon which requires various other scores [16]. The OBPS rates three colonic segments independently. Their scores are then summed at the end of the colonoscopic examination to give a total score. Furthermore, the OBPS includes an overall fluid quantity in the colon (rated o to 2) which allows subjective estimation of residual liquid. Thus, OBPS is a simple, objectively framed bowel preparation quality scale that assesses colonic segments individually and overall colonic fluid. It provides a summary score for the entire colon. This scale has high inter-observer reliability for total scoring and segmental colonic preparation quality [17]. The strength of our study was that we used two overall grading scales which included segmental scoring to validate the quality of the bowel preparation. Segmental scoring was conducted separately. Results supported the overall efficacy. Scores for the three colon sections were significantly higher in the OSS group than those in the AA + PEG group.

Adenoma detection rate is defined as the percentage of procedures per colonoscopist in which at least one adenoma is detected. It has been considered as a strong quality indicator for effective colonoscopy [19-22]. However, most patients enrolled in the present study had experience of colonoscopic examination or a prior history of colon polyp. We could not analyze polyp detection rate or adenoma detection rate for this reason. There was no difference between the two study groups regarding serious adverse events except for nausea and abdominal cramping which did not require further medical management. In the symptom questionnaire, the OSS group scored significantly higher for nausea and abdominal cramping compared to the AA + PEG group. Differences in taste among Asian subjects may cause higher scores for nausea than other Caucasian studies $[11,13,14]$. However, the degree of adverse symptoms in both groups was less than mild. There were no significant differences between the two groups for taste, stomach bloating, or sleep disturbance. These problems were also acceptable.

Our study has several limitations. First, we used a cleansing score that assessed three bowel segments. Recent studies have shown a more detailed evaluation that divides the colon into five or six segments $[7,23]$. However, both BBPS and OBPS scores have been extensively validated for general use in clinical studies [16]. Second, inter-investigator variation for bowel preparation scores unfortunately occurred in this investigation. Colonoscopists in two hospitals had no opportunity to reach a sufficient agreement about measurement of two bowel preparation scales. Third, AA + PEG bowel preparation adequacy scored less than that reported by other studies (88\% to 90\%) [7,24]. The small number of subjects enrolled in our study might have led to this discrepancy.

In summary, OSS provided a non-inferior efficacious bowel cleansing than AA + PEG regimen without compromising safety or tolerability. Bowel cleansing quality and patients' acceptance of the OSS formulation in Asian people indicate the potential of OSS as an alternative bowel cleansing method to AA + PEG for clinical use. Further prospective studies in larger populations are needed to confirm this finding.

\section{KEY MESSAGE}

1. Oral sulfate solution (OSS) is an emerging cleansing agent for bowel preparation in the world.

2. OSS is also an excellent efficacious bowel cleansing agent in Asian people.

3. Patients' acceptance was generally good in Asian people, although patients receiving OSS had more nausea and abdominal cramping than those receiving ascorbic acid plus polyethylene glycol. 


\section{Conflict of interest}

This research was supported by research fund of M.H. Healthcare.

\section{REFERENCES}

1. Dakkak M, Aziz K, Bennett JR. Short report: comparison of two orally administered bowel preparations for colonoscopy. Polyethylene glycol and sodium picosulphate. Aliment Pharmacol Ther 1992;6:513-519.

2. Hamilton D, Mulcahy D, Walsh D, et al. Sodium picosulphate compared with polyethylene glycol solution for large bowel lavage: a prospective randomised trial. Br J Clin Pract 1996;50:73-75.

3. Landreneau SW, Di Palma JA. Update on preparation for colonoscopy. Curr Gastroenterol Rep 2010;12:366-373.

4. Parra-Blanco A, Ruiz A, Alvarez-Lobos M, et al. Achieving the best bowel preparation for colonoscopy. World J Gastroenterol 2014;20:17709-17726.

5. Vemulapalli KC, Rex DK. Guidelines for an optimum screening colonoscopy. Curr Colorectal Cancer Rep 2012;8:6-15.

6. Enestvedt BK, Tofani C, Laine LA, Tierney A, Fennerty MB. 4-Liter split-dose polyethylene glycol is superior to other bowel preparations, based on systematic review and meta-analysis. Clin Gastroenterol Hepatol 2012;10:12251231.

7. Corporaal S, Kleibeuker JH, Koornstra JJ. Low-volume PEG plus ascorbic acid versus high-volume PEG as bowel preparation for colonoscopy. Scand J Gastroenterol 2010;45:1380-1386.

8. Kim SH, Kim JW. Low volume polyethylene glycol (PEG) plus ascorbic acid, a valid alternative to standard PEG. Gut Liver 2016;10:160-161.

9. Rees DC, Kelsey H, Richards JD. Acute haemolysis induced by high dose ascorbic acid in glucose-6-phosphate dehydrogenase deficiency. BMJ 1993;306:841-842.

10. Rex D, Di Palma J, McGowan J, Cleveland M. New oral sulfate solution provides superior overall and right colon cleansing versus a standard PEG and electrolyte preparation. Am J Gastroenterol 2009;104:S180-S181.

11. Di Palma JA, Rodriguez R, McGowan J, Cleveland Mv. A randomized clinical study evaluating the safety and efficacy of a new, reduced-volume, oral sulfate colon-cleansing preparation for colonoscopy. Am J Gastroenterol
2009;104:2275-2284.

12. Allende D, Elmessiry M, Hao W, et al. Inter-observer and intra-observer variability in the diagnosis of dysplasia in patients with inflammatory bowel disease: correlation of pathological and endoscopic findings. Colorectal Dis 2014;16:710-718.

13. Rex DK, Di Palma JA, Rodriguez R, McGowan J, Cleveland $M$. A randomized clinical study comparing reduced-volume oral sulfate solution with standard 4-liter sulfate-free electrolyte lavage solution as preparation for colonoscopy. Gastrointest Endosc 2010;72:328-336.

14. Katz PO, Rex DK, Epstein M, et al. A dual-action, low-volume bowel cleanser administered the day before colonoscopy: results from the SEE CLEAR II study. Am J Gastroenterol 2013;108:401-409.

15. Aihara H, Saito S, Ohya T, Tamai N, Kato T, Tajiri H. A pilot study using reduced-volume oral sulfate solution as a preparation for colonoscopy among a Japanese population. Int J Colorectal Dis 2013;28:83-87.

16. Lai EJ, Calderwood AH, Doros G, Fix OK, Jacobson BC. The Boston bowel preparation scale: a valid and reliable instrument for colonoscopy-oriented research. Gastrointest Endosc 2009;69(3 Pt 2):620-625.

17. Byrne MF. The curse of poor bowel preparation for colonoscopy. Am J Gastroenterol 2002;97:1587-1590.

18. Kang MS, Kim TO, Seo EH, et al. Comparison of the efficacy and tolerability between same-day picosulfate and split-dose polyethylene glycol bowel preparation for afternoon colonoscopy: a prospective, randomized, investigator-blinded trial. Intest Res 2014;12:53-59.

19. Millan MS, Gross P, Manilich E, Church JM. Adenoma detection rate: the real indicator of quality in colonoscopy. Dis Colon Rectum 2008;51:1217-1220.

20. Kaminski MF, Regula J, Kraszewska E, et al. Quality indicators for colonoscopy and the risk of interval cancer. $\mathrm{N}$ Engl J Med 2010;362:1795-1803.

21. Rex DK, Schoenfeld PS, Cohen J, et al. Quality indicators for colonoscopy. Gastrointest Endosc 2015;81:31-53.

22. Wang HS, Pisegna J, Modi R, et al. Adenoma detection rate is necessary but insufficient for distinguishing high versus low endoscopist performance. Gastrointest Endosc 2013;77:71-78.

23. Bitoun A, Ponchon T, Barthet M, et al. Results of a prospective randomised multicentre controlled trial comparing a new 2-L ascorbic acid plus polyethylene glycol and electrolyte solution vs. sodium phosphate solution in 
patients undergoing elective colonoscopy. Aliment Pharmacol Ther 2006;24:1631-1642.

24. Ell C, Fischbach W, Bronisch HJ, et al. Randomized trial of low-volume PEG solution versus standard PEG + electrolytes for bowel cleansing before colonoscopy. Am J Gastroenterol 2008;103:883-893. 